\title{
Arthralgia, CTCAE
}

National Cancer Institute

\section{Source}

National Cancer Institute. Arthralgia, CT CAE. NCI Thesaurus. Code C143298.

A disorder characterized by a sensation of marked discomfort in a joint. 